\title{
Development of a High-Temperature Smart Transducer Interface Node and Telemetry System (HSTINTS)
}

\section{CRADA Final Report}

\author{
CRADA No. ORNL98-0530 \\ Lockheed Martin Energy Systems Inc. and Halliburton Energy Services Inc.
}

November 3, 2006

\author{
Sponsored by \\ Halliburton Energy Services
}
Participant : $\quad$ Halliburton Energy Services Inc.
PI : John Minear / Roger Schultz
Address:
Phone:
Research Institution
ORNL
PI:
Mark Buckner / James Moore
Address:
P.O. Box 2008 MS 6006 Oak Ridge, TN 37831
Phone: (865) 574-7253




\begin{abstract}
Halliburton Energy Services and Oak Ridge National Laboratory established a CRADA to conduct applied research to develop a general purpose, High-Temperature, Smart Transducer Interface Node and Telemetry System (HSTTNTS) capable of temporallycoherent raultiple-channel, high-speed, high-resolution data transduction and acquisition while operating in a hostile thermal, chemical, and pressure environment for extended periods of time over a siggle coaxial cable. This ambitious, high-risk effort required development of custom dielectric isolated integrated circuits, amplified bybrid couplers for telemetry and an audio-frequency based power supply and distribution system using an engineered application of standing waves to compensate voltage drop along a 2 mile long cable. Several goals were achieved but underestimated challenges and a couple of mistakes hampered progress. When it was determined that an additional year of concerted effort would be required to complete the system demonstration, the sponsot withdrew funding and terminated the effort.
\end{abstract}

\title{
Statement of Objectives
}

The central objective of HSTINTS was to develop a telemetry backbone for future downhole instrumentation packages and temporally-coherent seismic data acquisition based on an emerging IEEE (P1451) smart transducer standard. Owing to system complexity and anticipated risks potential show stopping tasks were attacked first. Eight requirements were established:

1. Integration of IEEE-1451 standards based smart transducers, both sensors and actuators.

2. Oscillator, amplifier, mixer and other digital control circuitry for use with quartz temperature and pressure transducers.

3. Low-noise charge sensitive amplifier for use with 3-axis MEMS (piezo) accelerometers.

4. High resolution sigma-delta analog to digital converters

5. Data compression

6. Node level signal processing and actuator control

7. Robust telemetry utilizing multiplexed of channels for signal and power coupling Capable of handling at least $100 \mathrm{Mbits} / \mathrm{sec}$ effective data-rate Full-duplex communication Synchronous real-time (temporally coherent) data acquisition Single coaxial cable Multiple system clocks Option of implementing various communication protocols on top of the physical layer

8. $160^{\circ} \mathrm{C}$ continuous operation for at least 10 years 
Six tasks were established in pursuit of the objectives:

1. Demonstrate both high-temperature circuits and foundry process capable of $160^{\circ} \mathrm{C}$ continuous operation for 10 years; power and data transmission technique capable of meeting power and bandwidth requirements of HSTWNTS; telemetry protocol and high temperature circuitry for a quartz pressure and temperature transducer.

2. Prototype a "Smart Well" umbilical with five levels of pressure and temperature sensor nodes capable of $160^{\circ} \mathrm{C}$ continuous operation for 10 years.

3. Demonstrate high-temperature high performance signal processing and data compression circuitry for downhole " $4-0$ " seismic applications.

4. Prototype an umbilical with five levels of pressure and temperature sensor nodes and fifty levels of "4-D" seismic sensor nodes capable of $160^{\circ} \mathrm{C}$ continuous operation for 10 years.

5. Develop advanced downhole technologies and sensors for "Smart Well, 4D Seismic," and downhole video.

6. Design and development an intelligent global reservoir monitoring and control system.

\section{Benefits to the Funding DOE Offices Mission}

No DOE funds were expended on CRADA. \$232,980 of added factor and depreciation charges were waived in support of the project. DOE's ORNL benefited by having the technical staff develop experience and expertise in next generation leading edge high temperature integrated circuit design, and spread spectrum CDMA if transmission techniques, hardware, and the capabilities and limitations thereof.

\section{Technical Discussion of Work Performed by All Parties}

From the onset it was recognized and documented that the project requirements exceeded the capabilities of each institution individually and that close co-ordination and collaboration would be required in order to be successful. This was accomplished by weekly teleconferences and approximately quarterly meetings between the Halliburton and ORNL research teams. These discussions encompassed progress reports and technical discussion of what worked and what difficulties encountered. Collaborative exchanges formulating strategies and approaches for addressing problems both anticipated and uncovered were the usual agenda of these meetings.

Primary task leadership roles were assurned according to core competencies of the participants. ORNL led the communications protocol, if system, and electronics development while the Halliburton team led the development of the cable, water-tight connectors, downhole actuator controls and overall project priorities and direction. 
The primary challenges of HSINTS lay not with what the systern had to do but with the extreme environment, power, and size constraints dictated by the application. Both active and passive parts have difficulties operating at $160^{\circ} \mathrm{C}$ with a 10 year lifetime.

1. Since the system is immersed at high temperature and pressure in an electrolyte solution DC voltage gradients typical of common power distribution systems cannot be tolerated due to electrochemical corrosion. A power failure in a node represented not only a single point failure but negatively impacted the entire system. An electrically simple $A C$ power arrangement was required if 10 year operability was to be insured. A $/ 8$ system operating at $8 \mathrm{kHz}$ was used. Careful modeling confimed that with distributed loading the standing wave gain compensated the cable's resistive voltage drop within $10 \%$ along the entire 10,000 feet of the line. This eliminated the need for switching regulators in the nodes.

2. Several high temperature CMOS foundry processes were thoroughly evaluated for high temperature capabilities. A silicon on sapphire dielectric-isolated process was chosen for the project.

3. The system had very high dynamic range requirements which could not be met with analog modulation schemes, additionally space and power requirements severely limited the filtering that could be accomplished downhole. Whatever method chosen had to be integratable. CDMA was initially chosen, later it was proven that Synchronous CDMA would be required due to the number of channels used. Difficulty arose in the downhole frequency synthesizer primarily due to excess phase noise in a commercial SOS synthesizer. This problem would have necessitated a custom designed PLL chip if the project had been continued to completion.

4. A Sigma-Delta A/D modulator was designed and fabricated which provided 15 bit dyoamic range at $160^{\circ} \mathrm{C}$ and continued to function to well above $200^{\circ} \mathrm{C}$. A switched bit connection in the digital decimation filter required a second fabrication iteration with attendant delays and costs. The final layout corrected the error and the chip pinout was arranged in such a way that the chip set could be rounted and wirebonded in a single package. The project was terminated before final testing was accomplished but a number of useable wafers were fabricated by Halliburton

5. A high temperature operational amplifier was designed, fabricated, and tested. A patent is expected to issue in 2007 on this circuit.

6. An amplified hybrid coupler/line equalizer was developed to allow entrance and and exit of signals at nodes while leveling and equalizing the line. A simple repeater systern could not be used because a node power supply failure would create a single point system failure mode that would be inaccessible. Testing revealed that impedance continuity is a critical performance parameter in the feed forward coupler due to the reinforcement of reflected signals reducing the signal to interference ratio on the line.

7. A special cable design was required to meet demanding electrical, chemical and physical properties. The specifications required low attenuation in conjunction with small diameter and good impedance uniformity. The dielectric and jacket had to be impermeable to salt and acidic water while maintaining a low dielectric 
constant. Working with the Halliburton team WL Gore successfully fabricated a sample cable with appropriate properties to perform in the target environment.

8. During the course of the project IEEE 1451 failed to maintain sufficient industry support to allow the necessary commercial IC's executing the protocol to reach production. The protocol eventually had to be abandoned.

\section{Subject Inventions}

US Patent Application 20060049873 filed Sept 7, 2004, "Rail to rail differential input amplification stage with main and surrogate differential pairs".

\section{Commercialization Possibilities}

This being a $100 \%$ funds in CRADA, commercialization is controlled by Halliburton Energy Services. Lessons leamed in the research are applicable to ORNL's ongoing wireless technology projects and integrated circuit designs.

\section{Plans for Future Collaboration}

Halliburton Energy Services is not contemplating further collaboration in the foreseeable future.

\section{Conclusions}

The technical teaming and sharing of responsibilities between the ORNL and HES research teams was exemplary. The challenges were greater than anticipated. The project though not completed had a number of significant successes at the subtask level and those developments are available and applicable for a wide range of applications. The system complexity and $r$ expertise necessary to operate and troubleshoot a system of this type in the field probably makes this approach economically unfeasible at the current state of the art. 